\title{
DEVELOPMENT OF ISR FOR QUADCOPTER
}

\author{
Prabhjot Singh Sandhu' \\ ${ }^{1}$ Lovely Professional University, Phagwara, India
}

\begin{abstract}
Research organizations all over the world are finding and developing more and more application areas for UAV's. The prominent areas where UAV's have marked their presence include largely military, air force and upper atmosphere exploration ${ }^{[1]}$. Presently the technology involved in military application Quadcopter is inaccessible and secret, thus in this paper attempt has been made to develop such a UAV that has most of the functionality of a military grade UAV but can be constructed using off the shelf components. The emphasis is on development of low cost, reliable, accurate intelligence, surveillance and reconnaissance technology (ISR) ${ }^{[2]}$. The use of a specialized technology for control transmission will make it fail proof and snoop safe. The epilogue for the paper is a complete comparison of a low level and high level off shelf technology and the concerned development platform.
\end{abstract}

Keywords - ISR; UAV; quadcopter; ISR; multiwii; arduino; kk board; GPS

****

\section{INTRODUCTION}

ISR, an abbreviation for Intelligence, Surveillance and Reconnaissance is the most sought after technology of present era because of its strategic importance for countries, it is a safe guard of interest of one's own country by efficiently and secretly gathering data of the neighbor countries, although morally it would appear evil but it is a necessary evil ${ }^{[3]}$. Post and pre world war years saw an increase in insurgency of spies but a spy being a mortal asset for a country can never be compromised or if compromised proves fatal, so it was thought that ISR has to be done but not by spies but by machines. First attempts were land rovers which met great difficulties due to their inabilities to cope with unknown terrains, than came amphibious era which did not last for more two years due to their higher power requirements and low output ${ }^{[4]}$. All these difficulties with land and water based bots pointed to the last domain: airs, extensive research went into this domain and UAV's capable of ISR were developed.

Presently another requirement has popped up and is picking up pace with researchers, that is to make the ISR technology cheap and accessible so that its application areas can be increased. From this race a clear winner of UAV platform has emerged and that is a Quadcopter ${ }^{[5]}$. The Quadcopter with all its pros has become a platform of choice and ISR is now being developed keeping the Quadcopter as its centre. Quadcopters and other fixed wing aircrafts are now being exploited for their use in ISR. Fixed wing aircrafts unlike the others derive their thrust from individual motor assembly or a set of revolving narrow airfoils. The movement of the aircraft is based on the rotational speed of each of these airfoils; change of speed changes the position. The aircraft primarily is governed by control of the four major axes namely; pitch, roll and yaw.
Quadcopter consists of four narrow airfoils or lift generators located at the four ends of a perfect square shape. These lift generators are run by a very high speed motor and a precise balanced propellers, forcing the air flow down it generates the thrust to lift the Quadcopter above the surface ${ }^{[6]}$. Since the weight is shared by four independent motors that mean each motor has to lift one-fourth of the weight thus we can make motors small and small motor size gives us higher efficiency values.

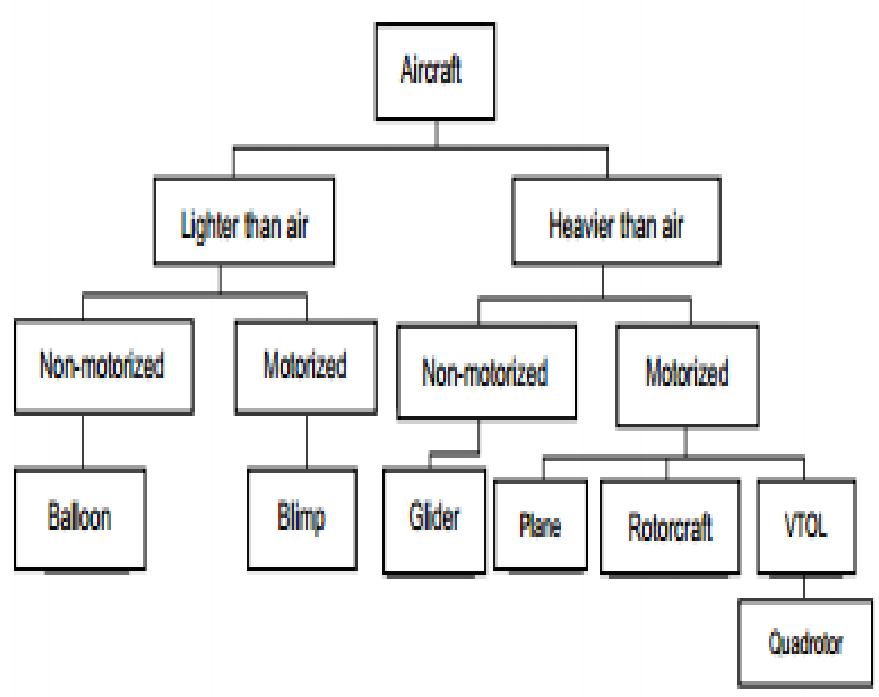

Fig 1: Aircraft classification based on flying principle 


\section{PHYSICAL PROPERTIES OF QUADCOPTER}

\subsection{Physical Principles of Aviation}

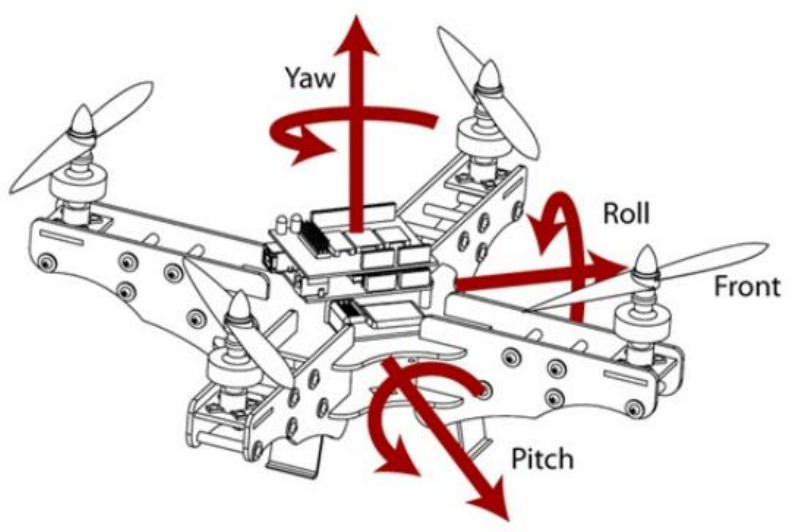

Fig 2: Axes of Quadcopter

For a successful flight it is very important to balance all the forces acting on the quadcopter only then will it be able to do things it is intended to do. At any point of time in space a number of forces are acting on the quadcopter on its three axes viz. roll, pitch and yaw; the overall resultant of these active forces decided the motion of the quadcopter. For a complete understanding of these forces we need a further detail of the three axes of the quadcopter (fig 2).

1) Yaw: It is the vertical axis that passes through the geometric centre of the quadcopter. Rotational force vector of all the four motors acts at the centre and cancels out each other at the exact geometric centre, in conditions when it does not cancel and the resultant vector has net positive or negative magnitude the quadcopter rotates about this axis clockwise or anti clockwise respectively.

2) Pitch: It is the axis that passes horizontally parallel to the plane of quadcopter extending towards the front and back end of the quadcopter. Rotational force vector of all the four motors acts at the centre and cancels out each other at the exact geometric centre, in conditions when the resultant of the rotational force vector is not zero but either positive or negative the quadcopter moves in the forward or backward direction respectively.

3) Roll: It is the axis that passes horizontally parallel to the plane of quadcopter extending from left to right. Rotational force vector of all the four motors acts at the centre and cancels out each other at the exact geometric centre, in conditions when the resultant rotational force vector is not zero but either positive or negative the quadcopter moves in the right or left direction respectively.

\subsection{Motion of the Quadcopter}

How the quadcopter moves can be answered by understanding very simple mechanism of unstable forces, suppose a system is stable at any point in the space at any given time such that all the forces acting on it are balanced. Now we change force in any one of the axes i.e. roll, pitch or yaw, the total force will no longer be stable and act towards a specific direction i.e. in the direction of the resultant force vector. Thus, the motion of quadcopter is achieved. Discussing this in detail for every axes (fig 3):

1) Up/Down: For this motion none of the axis is involved only the thrust that is generated by the propellers governs up/down motion. In simple physics more the thrust greater altitude it will achieve and vice-versa.

Thrust $\rightarrow$ Downward Force Generated by Propellers

2) 360 Degrees Rotation: The axis involved in this motion is the yaw axis, when the resultant of this axis is not zero and is pointing either in clockwise or anticlockwise direction then the copter also moves in that direction. This is done by increasing the thrust of two diagonally same motors and keeping the other diagonally same motors at same levels of thrust, than the direction in which the motors with increased thrust are moving the quadcopter will rotate in that direction.

3) Front/Back: The axis involved in this motion is the pitch axis, when the resultant of this axis is not zero and is pointing either in front or back direction then the quadcopter moves in that direction. This is done by increasing or decreasing the thrust of front and rear pair of motors. If we intend to move in the forward direction than the thrust of the back motors is increased and vice-versa

4) Left/Right: The axis involved in this motion is the roll axis, when the resultant of this axis is not zero and is pointing either in left or right direction then the quadcopter moves in that direction. This is done by increasing the thrust of either left or right pair of motors then the copter shifts in the opposite direction. If we intend to go in the left direction than the thrust of the right pairs is increased and vice-versa
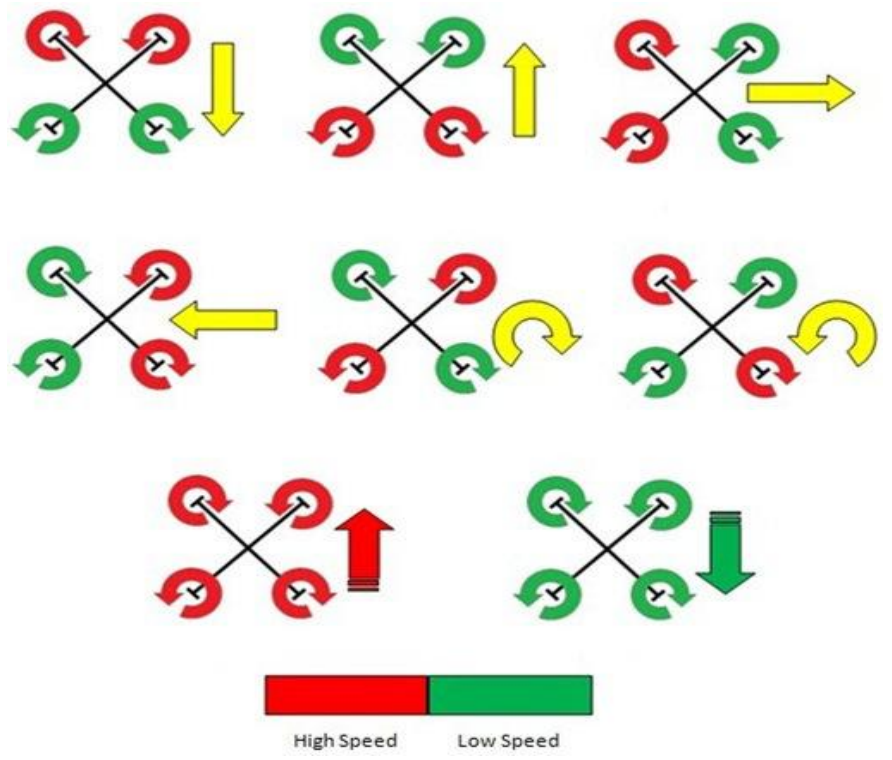

Fig 3: Movement depending on thrust change 


\subsection{Mathematical Analysis of Thrust}

Thrust generated by the propellers can be calculated by using the following technique:

$$
\mathrm{T}=\left(\pi \mathrm{D}^{2} \rho \mathrm{P}^{2}\right) / 2 \mathrm{~kg}
$$

$\mathrm{T}=$ Thrust of the propeller in grams

$\mathrm{D}=$ Diameter of the propeller in meters

$\rho=$ Density of air $\left(1.225 \mathrm{~kg} / \mathrm{m}^{3}\right)$

$\mathrm{P}=$ Power Multiplier

Power Multiplier can be calculated as:

$$
\mathrm{P}=\text { prop constant } *(\mathrm{rpm} / 100)^{\text {power factor }}
$$

Prop constant $=$ provided in the data sheet of product Power Factor $=$ provided in data sheet of product

\section{ELECTRICAL PARAMETERS QUADCOPTER}

For a quadcopter there are a number of electrical quantities involved those are very important in the selection process for the components of a quadcopter. Although they are very basic components required but they have a deeper impact on the quadcopters performance as the efficiency of the system is dependent on these. Practically an efficiency of over $80 \%$ is considered as an optimum value taking into account the resistance, heat and other loses.

\subsection{Mathematical Analysis}

Load: It is the total resistance attached with the battery i.e. the total resistance of the system.

Voltage: The voltage here is the voltage that is supplied by the battery usually measured in volts.

Current: It is the number of electrons flowing through the electrical system per unit time expressed in Amperes.

$$
\mathrm{I}=\mathrm{V} / \mathrm{R}(\mathrm{A})
$$

Electrical power: The optimum power available depending upon the input voltage and current.

$$
\mathrm{P}(\mathrm{E})=\mathrm{V} * \mathrm{I}(\mathrm{W})
$$

Mechanical power: The power available in actual use by the system. It is obtained by subtracting the losses incurred in the process of delivery of power.

$$
\mathrm{P}(\mathrm{M})=\mathrm{W} / \mathrm{T}(\mathrm{W})
$$

Work: It is the amount of the distance moved by the object when a unit force is applied on it.

$$
\mathrm{W}=\text { thrust } * \text { altitude }(\mathrm{J})
$$

Flight time: It is the time for which the quadcopter can be flown safely without depleting the battery.

$$
\mathrm{T}(\mathrm{F})=\mathrm{W} / \mathrm{P}(\mathrm{M})(\mathrm{s})
$$

Efficiency: It is the ratio of mechanical power to the electrical power.

$$
E(\eta)=P(M) / P(E)
$$

Internal Loses: The losses incurred due to internal resistances, heat dissipation and current leakage amount to internal loses.

\section{CONTROL SYSTEM}

For a successful and stable flight experience there are a lot of parameters like force acting on the three axes, external wind conditions etc those need to be taken care off. Earlier all this work had to be done by the pilot which was the prominent reason that it was not a choice of public but as the research has proceeded and advent of Fly By Wire technology the stability dynamics of the system are monitored and controlled by an independent system that works in conjunction to the user inputs. Now the flying experience for a quadcopter has improved many folds but it is still in infant stage and needs to mature a lot.

Fly By Wire (fig 4) technology represents a class of Control Systems specially developed for aerial vehicles which are not stable by design i.e. those whose designs do not comply the aerodynamics standards. For example the very famous B52 bomber aircraft of the US Air Force was developed across this platform as its design was not stable as per aerodynamics standards. Fly By Wire actually consists of sensing elements and a microcontroller that constantly monitors the values from the sensing elements and takes corrective action based upon an algorithm. The algorithm forms the brain of the section as it is the main function block that decides upon the corrective steps taken by the system to ensure the stability is maintained.

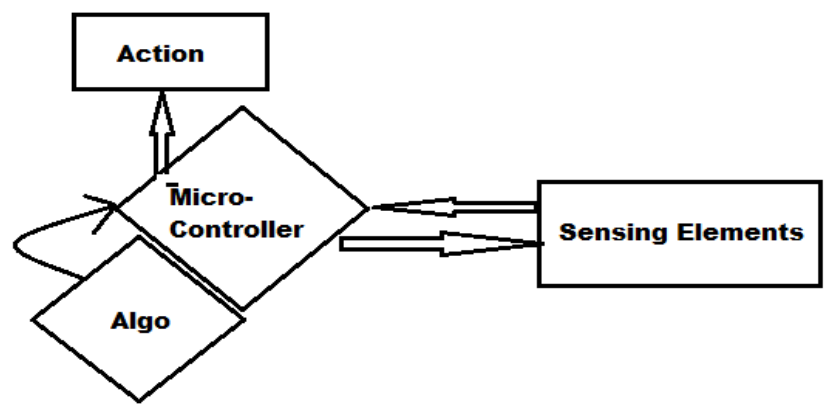

Fig 4: Block diagram of Fly by Wire 


\subsection{Sensing Elements}

\subsubsection{Inertial Measurement Unit}

This is special type of sensor that incorporates gyroscopes and accelerometers to determine the vehicles velocity and orientation. The major application of IMU is in the field of aerial vehicles where it is used for determination and control of vehicles position and motion. The class of vehicles where IMU's can be used is wide ranging from satellites in space, aero planes in sky to UAV's of military. IMU forms the core of the navigation system used in all the above listed applications. The process in which IMU first collects data and then calculates the position is called dead reckoning. The sensing can be capacitive, piezoelectric or any other. There are basically two types of IMU's 1. Crystal IMU's: These types of IMU's are based on a specific type of silicon called the Murata Crystals ${ }^{[12]}$. This is a synthetically modified Silicon structure which gives properties like lower internal inertia and vibration sensitivity. For each axis to be monitored a separate crystal has to be used making the overall construction of the system complex and prone to errors of time delay and accumulation. 2. MEMS IMU's: (Fig 5) With the recent advancement in the field of micro machining the MEMS based IMU's have developed as a popular choice due to inherent disadvantages of the Murata Crystals. Unlike Murata Crystals the MEMS based IMU do not exhibit high internal inertia or resonance ${ }^{[13]}$. In addition to this for MEMS internal dampers can be used effectively without decreasing the overall capability. MEMS IMU's are small in size and thus can be mounted easily, more over low pass filters and other filters may be added to the design to further improve upon the capability. In recent times motion processing technology has been incorporated into the available list of MEMS. Further MEMS IMU's may be based on various operating principles like capacitive, piezoelectric or resonance.
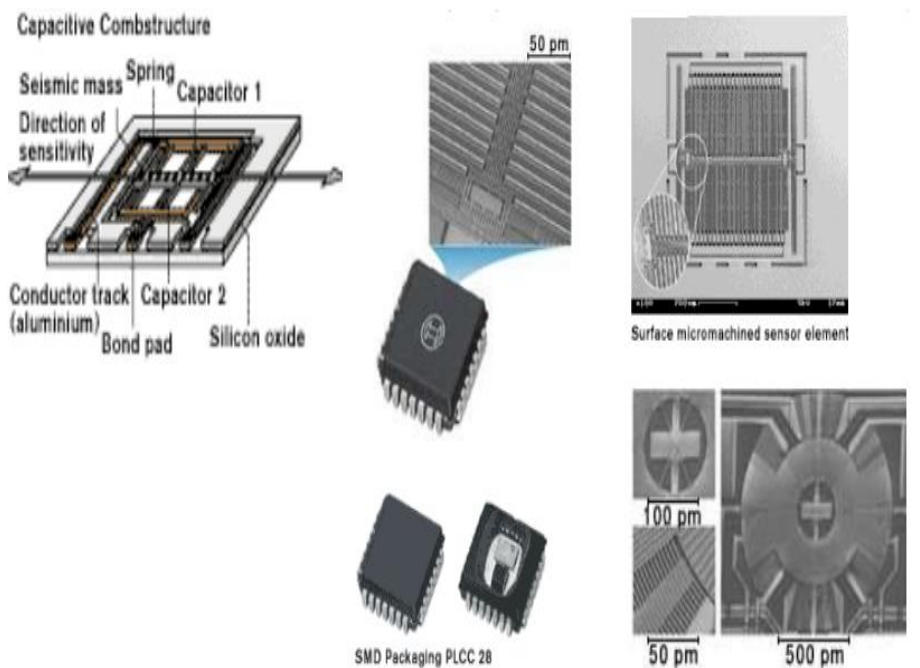

Fig 5: Capacitive MEMS IMU

\subsubsection{Barometric Pressure Sensor( Fig 6)}

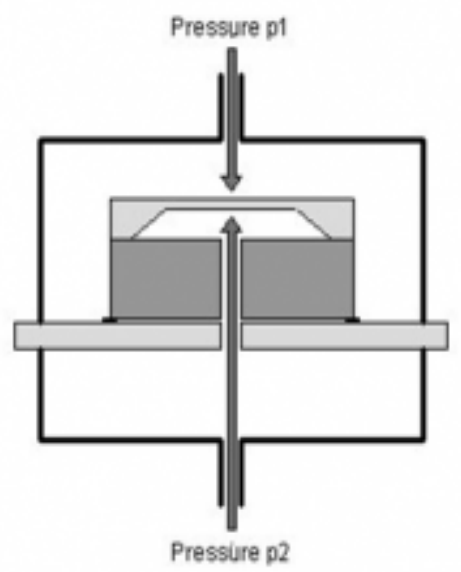

Fig 6: Physical Construction of a Differential Pressure sensor

In aerial application Pressure sensors are used for the determination of the altitude of the air craft. Since the vehicle has a 3 dimensional position vector it is very important to know the altitude of the vehicle to keep it on the right path and altitude. Barometric Pressure Sensor is a differential pressure sensor where the measurement of atmospheric pressure is made keeping in mind the pressure recorded at the ground level or zero altitude. Pressure is transduced through capacitor like structure in which the movable diaphragm compresses or expands depending upon the external pressure where as the air inside the diaphragms of the capacitive arrangement forms the dielectric. The change in pressure is recorded as a change in the capacitance of the device. As the aircraft ascends in air the pressure decreases. Barometric pressure sensor uses non linear logarithmic calibration as given:

$$
\mathrm{Z}=\mathrm{cT} \log \left(\mathrm{P}_{0} / \mathrm{P}\right)
$$

$\mathrm{c}=$ constant

$\mathrm{T}=$ absolute temperature

$\mathrm{P}=$ pressure at current altitude

$\mathrm{P}_{0}=$ pressure at sea level

The constant $\mathrm{c}$ is dependent on various factors like gravity, density of air etc. The calibration is such that the pressure is shown directly as an altitude above sea level.

Modern aircrafts make use of sensitive altimeter in which the sea level reference or ground reference can be set by the user. Such sensors calculate altitude by relation:

$$
\mathrm{H}=\left[1-\left(\mathrm{P} / \mathrm{P}_{\mathrm{ref}}\right)^{0.1902}\right] * 145366.45 \mathrm{ft}
$$

These sensors have an altitude resolution of less than $1 \mathrm{~m}$ which is very good compared to $20 \mathrm{~m}$ of GPS. 


\subsubsection{Magnetometer}

Magnetometers are used to find the direction of magnetic field at a point, strength of magnetic field and magnetization. There are various types of magnetometers depending upon their area of application. Magnetometers used in aerial vehicles are vector magnetometers. Vector Magnetometers electronically measure the components of electric field. They use three orthogonal magnetometers for the measurement of azimuth and dip. The type of measurement technique used is magneto resistive measuring. They are made of very thin strips of a permanent magnetic alloy of NiFe also known as magnetic film. The electrical resistance of the alloy changes with the change in electric field. They have a sharp axis of sensitivity of operation and can produce $3 \mathrm{D}$ versions. The response time is particularly low as compared to others which is less than 1 microsecond. The frequency of measurement can be scaled upto $1000 \mathrm{~Hz}$ which is phenomenal. These can effectively read between degrees i.e. its resolution can be in minutes and seconds. Mathematically magnetic field can be calculated as follows:

$$
\phi_{\mathrm{H}}=\mathrm{R}_{0}-\mathrm{R}_{\mathrm{H}} / \mathrm{R}_{\mathrm{H}}
$$

$\phi_{\mathrm{H}}=$ Current magnetic field

$\mathrm{R}_{0}=$ resistance at zero magnetic field

$\mathrm{R}_{\mathrm{H}}=$ resistance at a given magnetic field

\subsubsection{Global Positioning System}

Satellites above the earth broadcast there location and current time and the receivers listen to several satellites determining there time and location.

The satellite broadcast consists of two signals consisting of carrier waves which undergo phase change in a defined pattern at a precise rate and defined time. The receiver creates a replica of the phase change pattern, than shifts it in the time domain, advancing and delaying so that it may correlate it with the received signal. On successful correlation of the signals the correlator circuit will produce a large output which tells that lock-on to satellite is made. By determining how much the internal signal has been shifted in time tells the receiver when the broadcast was received. After the receiver determines how the internal clock was adjusted with respect to the GPS time it calculates how much time did it take for the broadcast signal to reach the receiver. Than the receiver multiplies this time with speed of light it finds its distance from the satellite.

The broadcast signal along with transmission of a specific phasechange pattern that is unique for a satellite also transmits additional data appended to the signal. The additional data forms the Navigation Message. The navigation message comprises of the current time to the nearest second and information required to calculate the position of the satellite at the time of transmission. Using this information the receiver then calibrates its time to the nearest second and computes the position of satellite. The receiver than calculates its position on the surface of an imaginary sphere whose centre is the satellite and radius as distance from satellite. The two frequencies on which all the satellites transmit are almost the same. The primary signal is broadcast on $1575.42 \mathrm{MHz}$ and this is called the L1 frequency. The technique used for the transmission is spread spectrum that allows to transmit many signals on the same frequency. L1 signal is modulated with two information signals known as the C/A (Coarse and Acquisition code) and P (Precise code). Another copy of the $\mathrm{P}$ code is transmitted on the other frequency called the L2 frequency, $1227.60 \mathrm{MHz}$ (fig 8).

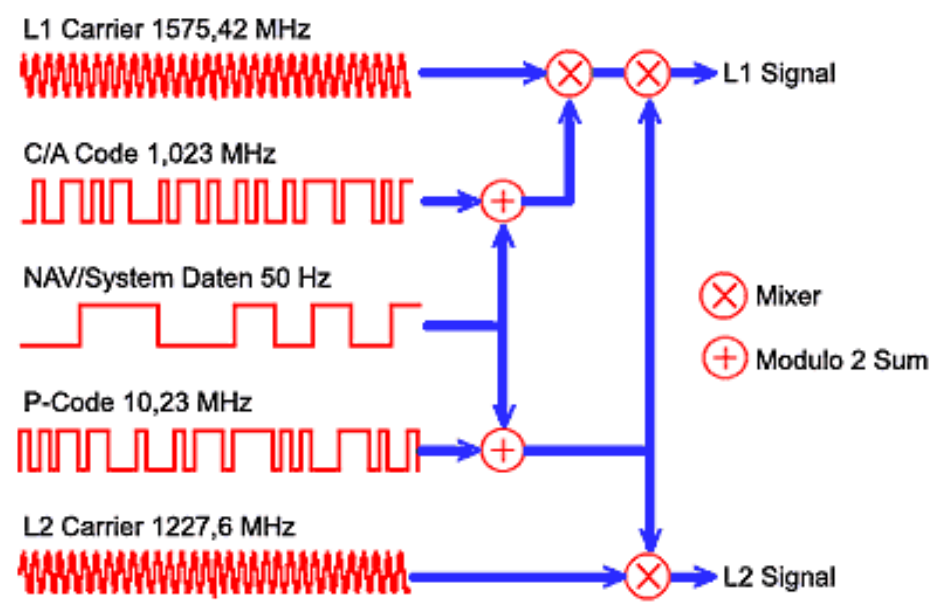

Fig 7: Signal broadcast from satellite

\subsubsection{PID Control}

Proportional-Integral-Derivative controller is a type of feedback based control system that is most widely used in automation processed. The algorithm of a PID controller revolves around the three separate constant parameters and is sometimes referred as three term control. Independently P denotes the present error, I denotes accumulation of past errors and $\mathrm{D}$ is the prediction of future errors. The weighted sum that is generated from these terms is then used to adjust the deviation in the process. Thus PID controller is an error based system which works by minimizing the error between measured process and a desired set point.

The three terms of the PID contribute to the manipulated variable (MV) i.e. the process to be controlled. The individual components are than added to find output of the PID. If $u(t)$ is the controller output than

$$
\mathrm{u}(\mathrm{t})=\mathrm{MV}(\mathrm{t})=\mathrm{K}_{\mathrm{P}} \mathrm{e}(\mathrm{t})+\mathrm{K}_{\mathrm{i}} \int_{0}{ }^{\mathrm{t}} \mathrm{e}(\tau) \mathrm{d} \tau+\mathrm{K}_{\mathrm{d}}(\mathrm{d} \mathrm{e}(\mathrm{t}) / \mathrm{dt})(12)
$$

$\mathrm{K}_{\mathrm{P}}=$ Proportional gain, a tuning parameter

$\mathrm{e}=$ Error

$\mathrm{K}_{\mathrm{i}}=$ Integral gain, a tuning parameter

$\mathrm{K}_{\mathrm{d}}=$ Derivative gain, a tuning parameter

$\mathrm{T}=$ Time or instantaneous time (the present) 
Analyzing the three terms independently:

1. Proportional term: The output of this term produces an output that is proportional to the current error. The response time is adjusted by multiplying the error with the constant gain term, given by:

$$
\mathrm{P}=\mathrm{K}_{\mathrm{P}} \mathrm{e}(\mathrm{t})
$$

This means that a higher gain term will produce a large change in the output for a given change. If the gain is too high the system may overshoot or if the gain is too low the system may become non responsive.

2. Integral term: This specifies the duration for which the errors are recorded before an action is taken. Mathematically it is the sum of instantaneous error over time and gives accumulated offset that should have been corrected.

$$
I=K_{i} \int_{0}{ }^{t} e(\tau) d \tau
$$

The integral gain if increased will decrease the time taken to attain set point and vice versa. However a very gain will lead to oscillations in the system.

3. Derivative term: This specifies the force with which the system responds to reach the set point from the given state of error.

$$
\mathrm{D}=\mathrm{K}_{\mathrm{d}}(\mathrm{d} \mathrm{e}(\mathrm{t}) / \mathrm{dt})
$$

This actually predicts the system position and thus reduces the settling time of the system. If the error in system is very large than derivative term becomes erratic and will destabilize the system. If the gain term is set too high than it may over shoot the system which can be controlled using a low pass filter.

The major problem that the user faces is the way to adjust or tune the parameters of PID to obtain the set point. A simple and most celebrated method of PID tuning is Ziegler-Nicolas (fig 8) method in which the gain is increased till the point the system begins to oscillate and then it is reduced. The entire process can be broken down into three easy steps which are:

1. Put the $K_{i}$ and $K_{d}$ terms as zero and then increase the $K_{P}$ term till the system begins to oscillate $K_{u}$, than multiply the $\mathrm{K}_{\mathrm{u}}$ factor by 0.50 to obtain $\mathrm{K}_{\mathrm{p}}$.

2. Calculate the $I$ factor by dividing $K_{p}$ by $P_{u}$ which is the oscillation period and then multiply it by 1.2 .

3. Take the product of $\mathrm{K}_{\mathrm{p}}$ and $\mathrm{P}_{\mathrm{u}}$ and divide by eight to obtain $\mathrm{K}_{\mathrm{d}}$.

\begin{tabular}{c|c|c|c|c|c|} 
Parameter & Rise time & Overshoot & Settling time & Steady-state error & Stability \\
\hline$K_{p}$ & Decrease & Increase & Small change & Decrease & Degrade \\
\hline$K_{i}$ & Decrease & Increase & Increase & Eliminate & Degrade \\
\hline$K_{d}$ & Minor change & Decrease & Decrease & No effect in theory & Improve if $K_{d}$ small
\end{tabular}

Fig 9: Effects of PID

\section{CONTROL BOARDS}

The control boards available for quadcopters are based on three available firmware's kk, multiwii, megapirate; these firmware's are based on an open source code available in the Google code directory. In this paper two boards one from kk and other from multiwii and megapirate domain were selected and comparisons were drawn.

1) $\boldsymbol{k} \boldsymbol{k}$ board: This board is based on murata gyros and has no other sensor on board. It has an Atmel 128p micro controller, and can support six speed controllers by taking direct input from the receiver. The flashing can be done by using an FTDI cable using the kk flash tool available on internet. Absence of many of the important sensors like pressure sensor, magnetometer etc although makes it simple but at the same time the stability of the quadcopter is also compromised, also if one wishes to add more sensors there is no option for the same. The flight of the quadcopter is not ideal and as such it does not hover rather it keeps moving in a certain direction depending upon the resultant force. The PID tuning of the copter is also not perfect rather than digital tuning analog potentiometers are used for tuning the PID which results in poor performance. Overall this board can be used only where precision is not required i.e. one has to scan an area on a random note.

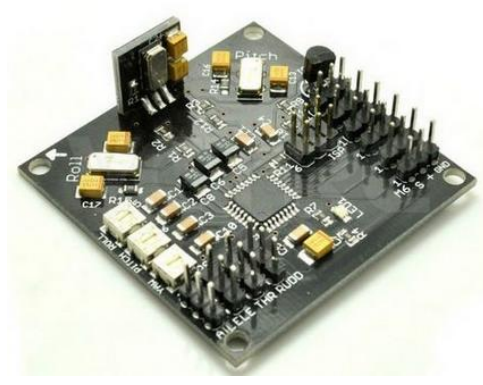

Fig 10: KK Board

2) Crius aiop(fig 11): This board is specially designed keeping in mind precision control and flexibility of adding more sensors. The board contains a high precision ATMega 2560 Microcontroller ,MPU6050 6 axis gyro/accel with Motion 
Processing Unit, HMC5883L 3-axis digital magnetometer, MS5611-01BA01 high precision altimeter, FT232RQ USBUART chip and Micro USB receptacle for in system programming. The board can be loaded with either multiwii or megapirate code making it all the more versaltile. GPS controller can be added for map based missions which is a big miss in kk boards. Overall this board is best suited for precision flying and thus is the choice for developing ISR Quadcopter.

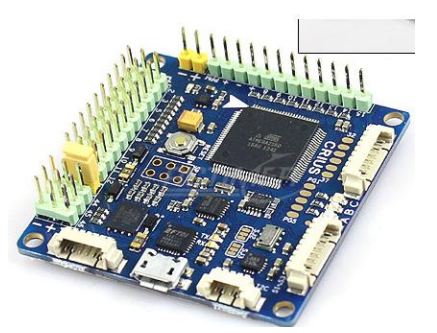

Fig 11: Crius aiop

\section{DEVELOPING ISR FOR QUADCOPTER}

1) Hardware: The base of the quadcopter can be a fiber glass or carbon fiber material as both these materials help to absorb vibrations caused due to the motors or turbulent atmosphere.

2) Electronics: The required motor size can be calculated based on the total model weight and then using (1) for the calculation of thrust, only brushless outrunner motors are used as they are small and light weight. The current of the ESC can be calculated from the voltage of the battery and the internal resistance of the motors. Ideally $1-2 \mathrm{~A}$ extra ability is maintained for very high speed operations. The battery used is a 3 cell Lithium Polymer battery with discharge rate greater than $25 \mathrm{C}$ so that the system may get the required current at all times. Lithium Polymer batteries are selected due to their lower weight to power ratio. The control board selected is Crius aiop due to its versatility. GPS is a UBLOX GPS unit based on a mediatek controller.

3)Tx \& $\boldsymbol{R} \boldsymbol{x}$ : The method for transmission of the control signal is selected so as to make it fail safe and snoop safe and to achieve this frequency hopping spread spectrum technique is used for transmitting the control signal. The receiver has bi orthogonal antennas so that the reception is of optimum quality with least amount of bit error rate.

The crius aiop is flashed with the latest multiwii code that has been changed for the specific components and use.

\section{EXPERIMENTAL RESULTS}

The initial flight test using kk board proved that this board cannot be used for precision applications and thus was discarded in initial stages of development.
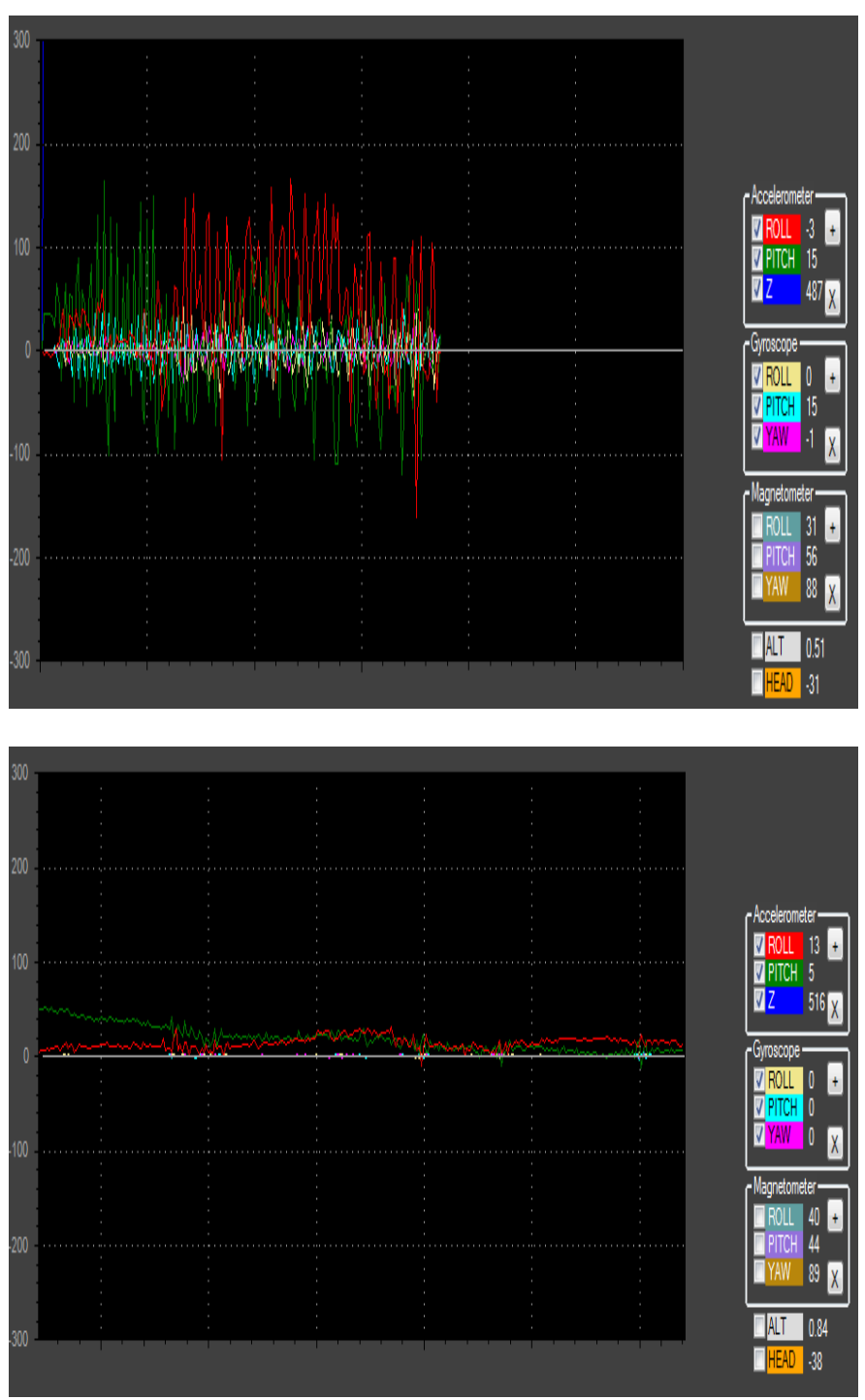

Fig 12: Vibration graph KK(top) Crius (bottom)

Fig 12 clearly show the in flight stability of the crius board as compared to the kk board.

The crius aiop was made the platform of choice for developing ISR system, multiwii is used instead of megapirate as it is more refined and better support is available in case things go wrong. The multiwii system has a very interactive graphical user interface that can be assessed in flight using telemetry for long distance and Bluetooth for short distance flights. The GUI (fig 13) gives real time information of the quadcopter performance, battery level, position on map and other necessary details which are not provided by the kk board and the megapirate system. 


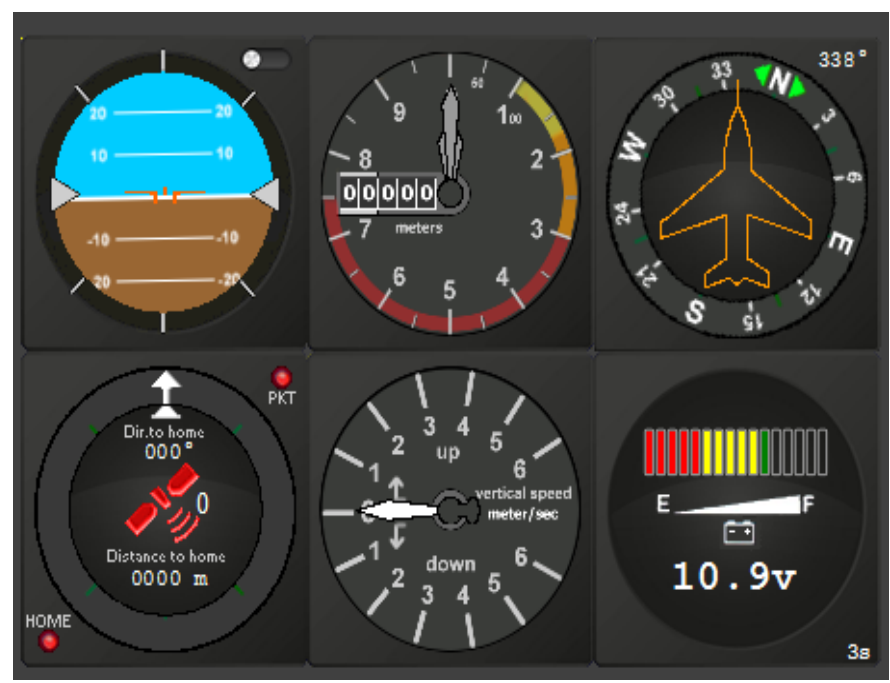

Fig 13: Real time data from quadcopter

Our experiment with GPS baud rates helped us to establish the fact that using a lower baud rate of 9600 gives a very low error percentage of $+/-2$ where as if the baud rates are increased the error in position can be of the magnitude of $+/-10$. The results were confirmed by the GUI.

The multiwii system has a mobile option i.e. we can use an android based smart phone to control the quadcopter. The application for the android gives the user real time data as well ability to upload new missions.

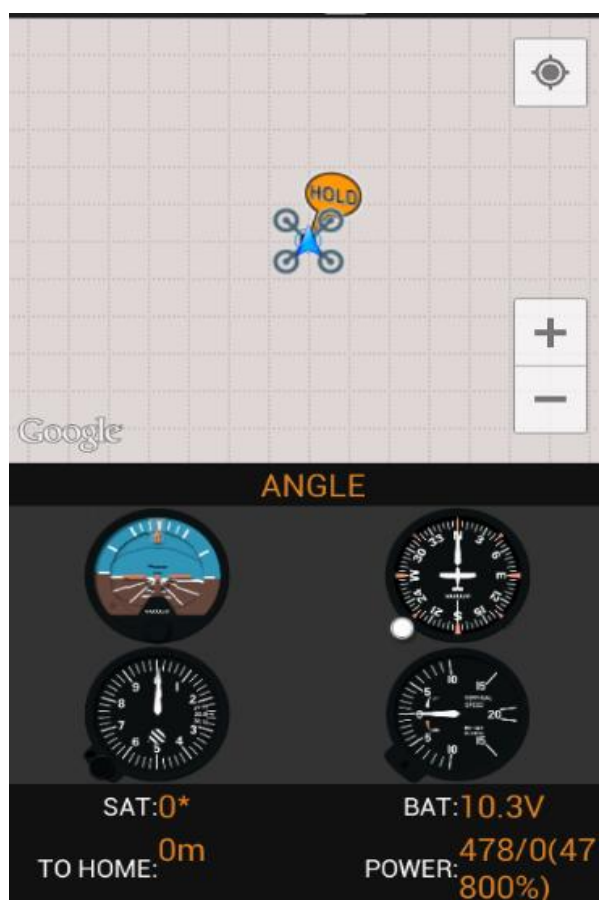

Fig 14: Mobile interface of multiwii
The multiwii system has a mission option with which user can mark waypoints on the map and create an autonomous path to be followed by the quadcopter.

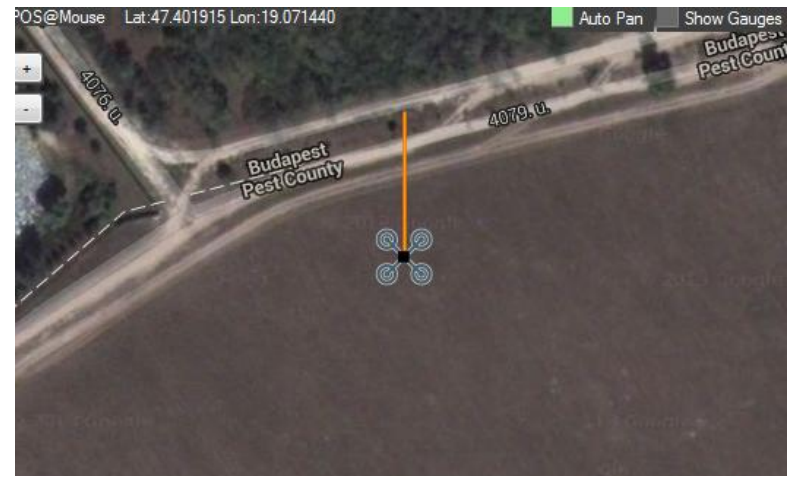

Fig 15: Mission Tab of multiwii

Our experiments with the low pass filter of the MEMS gyro helped us to conclude that $40 \mathrm{hz}$ refresh rate is best suited for middle sized quadcopters rather than $20 \mathrm{hz}$ which is better for bigger sized quadcopters.

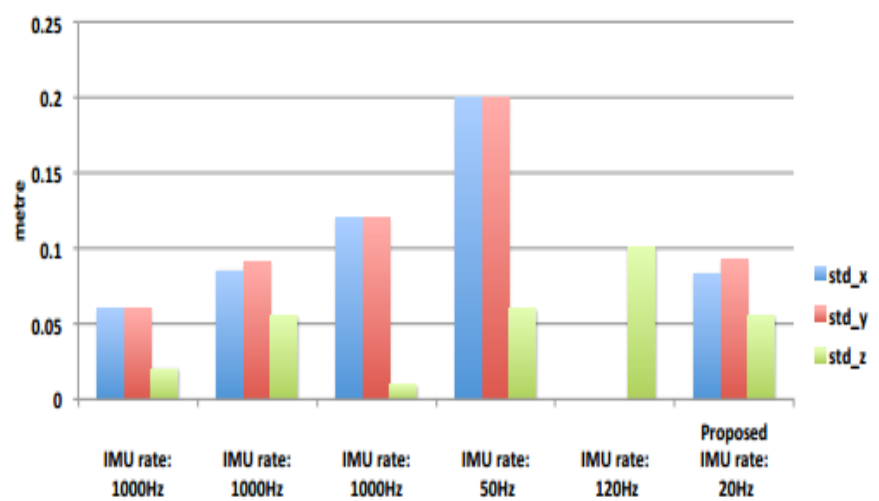

Fig 16: Statistical analysis of stability at different LPF

\section{CONCLUSIONS}

Our research work yielded a successful development of ISR system based on quadcopter platform that is inexpensive and can be made from off the shelf components. The use of semi autonomous option through maps broadens the application area of the developed system. The live video transmission available from the quadcopter can be utilized to generate a $2 \mathrm{D}$ map of the area using MATLAB, and overlaying two maps made at different times can help to trace changes that have taken place in the area.

The developed platform can be used as a low cost alternative to carry out explosive detonation of army targets with very high level of precision. 
The future research can be carried out to implement SWARM technology so that a fleet of quadcopters can be sent that communicate with each other and perform required operations.

Furthermore, future research in area of vibration resistant IMU sensors will result in quadcopters with resolution of $1 \mathrm{~cm}$ against the present standard of $10 \mathrm{~cm}$.

\section{ACKNOWLEDGMENTS}

The author would like to thank EZIO and EOS BANDI developers for their support and technical assistance. The author would also acknowledge Asst. Prof Swapnil Bhagwari who with his abundant knowledge of programming and other skills was greatly helpful at every step.

\section{REFERENCES}

[1]. "Designing a Spatially Aware and Autonomous Quadcopter" Michael Y. Chen, Derrick H. Edwards, Erin L, Boehmer, Nathan M. Eller IEEE 2013, Conference

[2]. "A Survey of Quadrotor Unmanned Aerial Vehicles" Shweta Gupte, Paul Infant Teenu Mohandas, James M. Conrad, University of California IEEE 2012, November

[3]. "The Use of UAVS in USA's Security by an Engineering Education Approach"Eduardo I. Ortiz-Rivera, Angelina Estela, Carlos Romero, Jesus A. Valentin, University of Puerto Rico IEEE 2012, July

[4]. "Visual Tracking and Control Of a Quadcopter" Markus Achtelik, Tianguang Zhang, Kolja Kuhnlenz, Martin Buss, University of Germany IEEE 2009

[5]. "Model Simplification of an Unmanned Aerial Vehicle" Ioan-Radu Morar, Ioan Nascu, Technical University of ClujNapoca IEEE 2013

[6]. "Design and Construction of RC Quadcopter based on STC12C5624AD" Stevie Jeremia, Julinda Pangaribuan, University of Pelita Harapan IEEE 2012, Conference

[7]. "Quadcopter Design and Implementation as a Multidisciplinary Engineering Course" Igor Gaponov, Anastasia Razinkova, Korea University of Technology and Education IEEE 2013, Conference

[8]. "A simple visual navigation system for a UAV" Tomas Krajnik, Matias Nitsche, Sol Pedre, University of Prague IEEE 2012, Conference

[9]. Control System by: BS Manke

[10]. www.eosbandi.com/downloads

[11]. www.rcbazaar.com

[12]. www.rcbharat.com

[13]. www.thehobbyshop.in 